\title{
Comportamento infantil, rotinas alimentares e de higiene, e queixas odontológicas de pacientes infantis durante a pandemia da COVID-19
}

\section{Behaviour, eating habits, hygiene routines, and dental complaints among paediatric patients during the COVID-19 pandemic}

\author{
Isabelle de Melo Xavier Bentinho' ${ }^{1}$, Cintia Regina Tornisiello Katz ${ }^{*}$
}

\begin{abstract}
RESUMO
Este estudo objetivou avaliar os efeitos do isolamento social no comportamento infantil, nas rotinas alimentares e de higiene, e na saúde bucal de pacientes infantis durante a pandemia da COVID-19. Realizou-se um estudo transversal, descritivo, entre dezembro (2020) a março (2021), por meio pesquisa online com pais e/ou responsáveis pelas crianças de 03 e 12 anos, das clínicas do Curso de Odontologia da Universidade Federal de Pernambuco. Os percentuais de crianças que apresentaram alterações comportamentais, nas rotinas alimentares e de higiene bucal foram, respectivamente, 90,0\%, 83,3\% e $75,0 \%$. As queixas odontológicas mais frequentes foram cárie e necessidade de extrações dentárias $(77,1 \%)$ e dor $(30,8 \%)$. Para $45,0 \%$ dos responsáveis, não haverá mudanças no comportamento infantil na oportunidade de retorno aos atendimentos odontológicos, e para $29,2 \%$ haverá maior colaboração. O período de isolamento social, implicou em mudanças comportamentais, de hábitos alimentares e de higiene bucal para grande maioria das crianças. Tais alterações merecem atenção dos profissionais de saúde na preparação das estratégias de atendimento às novas demandas da população infantil decorrentes do prolongamento do período da pandemia da COVID-19.
\end{abstract}

Palavras-chave: Pandemia; Comportamento; Crianças; Rotinas Alimentares; Saúde Bucal.

\begin{abstract}
This study aime to assess the effects of social isolation on behaviour, eating habits, hygiene routines, and oral health on paediatric patients during the COVID-19 pandemic. A cross-sectional study was conducted between December (2020) and March (2021), involving an online survey with parents/guardians of children three to 12 years of age in care at the clinics of the dentistry course of Universidade Federal de Pernambuco, Brazil. The percentages of children with changes in their behaviour, eating habits, and oral hygiene routine were respectively $90.0 \%, 83.3 \%$, and $75.0 \%$. The most frequent dental complaints were caries/the need for tooth extraction $(77.1 \%)$ and dental pain $(30.8 \%)$. For $45.0 \%$ parents/guardians, there will be no changes in children's behavior in the opportunity to return to dental care, and for $29.2 \%$ there will be collaboration. The period of social isolation involves changes in behaviour, eating habits, and oral hygiene for the majority of the sample studied. These aspects require the attention of health care providers regarding the preparation of strategies to meet new care needs resulting from the long COVID-19 pandemic.
\end{abstract}

Keywords: Pandemic; Behaviour; Children; Eating Routines; Oral Health.

\footnotetext{
${ }^{1}$ Universidade Federal de Pernambuco

*E-mail: cintia.katz@ufpe.br
} 


\section{INTRODUÇÃO}

Em dezembro de 2019, um novo tipo de coronavírus foi identificado na cidade de Wuhan, China, em pacientes com pneumonia de origem desconhecida. Devido a sua propagação violenta em todo o mundo, em março de 2020 a Organização Mundial da Saúde (2020) declarou a pandemia da COVID-19. Para conter a disseminação do vírus, no Brasil, o Ministério da Saúde recomendou ações preventivas como a lavagem frequente das mãos com água e sabão, uso de máscaras, além de evitar o contato próximo com pessoas sintomáticas. Foram implementadas medidas como o isolamento e/ou distanciamento social, restringindo a circulação de pessoas em locais que poderiam provocar aglomeração de pessoas (AQUINO, et al. 2020); muito embora essas medidas tenham sido adotadas de maneira dessincronizada entre os vários estados e municípios do país.

As medidas de prevenção à COVID-19 estenderam-se também às crianças, uma vez que, mesmo sendo pouco afetadas pelo quadro mais severo da doença, elas são potencialmente transmissoras do vírus (DONG, et al. 2020). Com isso, assim como os adultos, este grupo etário, também sofreu os efeitos das medidas restritivas, principalmente com o isolamento social.

Com o fechamento das escolas durante o período chamado de quarentena, isolamento rígido ou Lockdown, as crianças tiveram que passar mais tempo em casa, necessitando enfrentar uma nova e desafiadora rotina (CAMPAGNARO, et al. 2020). O uso de máscaras pelas crianças pode estar associado a dificuldades e desconfortos (ESPOSITO e PRINCIPI, 2020). O hábito de lavagem frequente das mãos ou a falta de orientação sobre a lavagem correta das mãos são ações que, a depender da idade, podem ainda não ter sido desenvolvidas (CHEN, et al. 2020).

O isolamento social pode trazer impactos negativos no comportamento e na saúde mental das crianças. Algumas alterações comportamentais como dificuldades de concentração, irritabilidade, medo, inquietação, tédio, sensação de solidão, ansiedade, alterações no padrão de sono e na alimentação podem ser manifestadas frente às adversidades do atual cenário (JIAO, et al. 2020)

Novas rotinas associadas ao período da pandemia podem implicar em mudanças nos hábitos alimentares e nas rotinas de higiene das crianças, aumentando o risco de doenças (LUZZI, et al. 2021). A alimentação inadequada pode aumentar a suscetibilidade ao desenvolvimento de doenças como obesidade, diabetes do tipo II e cárie dentária. 
Nesta última, alterações na rotina de higiene bucal podem resultar em um aumento significativo da sua prevalência (CAMPAGNARO, et al. 2020).

Em pesquisa publicada pela Universidade Federal de Pelotas (CHISINI, et al. 2021), após o primeiro caso de COVID-19 no Brasil, houve redução de $66 \%$ nos atendimentos odontológicos às crianças. Desta forma, considera-se que a interrupção dos atendimentos por um período tão longo certamente trará impactos negativos à saúde bucal da população infantil (LUZZI, et al. 2021).

Pelo exposto, a realização deste trabalho justifica-se pela necessidade de avaliar os efeitos do isolamento social no comportamento infantil, nas rotinas alimentares e de higiene, e na saúde bucal de pacientes infantis durante a pandemia da COVID-19, sendo este o objetivo principal deste estudo.

\section{MATERIAIS E MÉTODOS}

Foi realizado um estudo transversal por meio de pesquisa online com pais e/ou responsáveis pelas crianças atendidas nas clínicas do Curso de Odontologia da Universidade Federal Pernambuco (UFPE) - Brasil. A amostra foi composta por crianças entre 3 e 12 anos de idade que foram atendidas nas Clínicas Odontológicas do Departamento de Clínica e Odontologia Preventiva da UFPE durante o segundo semestre de 2018 e no primeiro e segundo semestres de 2019. Foram enviadas mensagens por meio de aplicativo de celular para 507 pais ou responsáveis, entre os meses de dezembro de 2020 e março de 2021, convidando-os a participar da pesquisa.

Foi critério de inclusão na pesquisa: ser pai/mãe ou responsável legal de crianças com idades entre 3 e 12 anos atendidas nas clínicas do Curso de Odontologia no segundo semestre de 2018 e no primeiro e segundo semestres de 2019; e concordar em responder a um formulário eletrônico (Google Forms), encaminhado por meio de aplicativo de celular, após contato telefônico prévio. Foi estabelecido como critério de exclusão a impossibilidade de responder às questões relativas à criança.

Os dados foram coletados por meio do preenchimento de um formulário online, encaminhado por aplicativo de celular aos pais/responsáveis, de acordo com os números telefônicos cedidos pelo Núcleo de Acolhimento e Pronto Atendimento do Curso de Odontologia da UFPE. Previamente ao envio do formulário, os responsáveis recebiam uma mensagem de texto com o convite para participação na pesquisa e explicações breves 
sobre o objetivo da pesquisa. Em seguida era enviado um arquivo contendo o Termo de Consentimento Livre e Esclarecido e o link de acesso ao formulário da pesquisa. Ao ler o termo e concordar em participar da pesquisa, o voluntário acessava o link do formulário. Ao abrir o formulário, era solicitado o preenchimento do número telefônico e após escolher a opção "concordar em participar da pesquisa", o questionário era liberado para ser respondido. O formulário foi encaminhado mais de uma vez para os responsáveis que mencionavam que tinham mais de um filho atendido nas clínicas do curso de odontologia e que pertenciam à faixa etária da pesquisa.

O formulário foi composto por 28 perguntas e todas se refeririam ao período da quarentena ou isolamento rígido realizado no estado de Pernambuco durante aos primeiros meses da pandemia da COVID-19 no ano de 2020. A análise dos resultados foi feita de forma descritiva, por meio dos dados fornecidos automaticamente pelo aplicativo Google Forms. Através desta ferramenta, foram obtidas distribuições absolutas e percentuais das variáveis estudadas que foram apresentadas no formato de tabelas. Esta pesquisa recebeu aprovação do Comitê de Ética em pesquisa da Universidade Federal de Pernambuco - Campus Recife (Parecer: 4.330.946; CAAE: 37278220.4.0000.5208).

\section{RESULTADOS}

Foram recebidos 132 formulários preenchidos. No entanto, doze formulários foram excluídos, pois se referiam a dados de crianças fora da faixa etária da pesquisa. Desta forma, a amostra final totalizou em 120 formulários respondidos por 115 responsáveis, sendo 100 mães, 11 pais e 4 avós que moravam com a criança.

A Tabela 1 mostra a distribuição da amostra de acordo com a idade, sexo e percepção dos responsáveis de acordo com a infecção pela COVID-19. A maioria das crianças tinham entre 7 a 10 anos de idade e era do sexo feminino. A média das idades das crianças foi 8,5 anos. Apenas 5,0\% crianças tiveram COVID-19 no período do estudo e 42,5\% tinham parentes ou pessoas próximas que tiveram COVID-19. 
Tabela 1 - Distribuição da amostra de acordo com a idade, sexo e percepção dos responsáveis de sobre a ocorrência da COVID-19

\begin{tabular}{|c|c|c|c|c|c|}
\hline Variável & $\mathbf{n}$ & $\%$ & Variável & $\mathbf{n}$ & $\%$ \\
\hline Faixa etária (anos) & & & Sexo & & \\
\hline 3 a 6 & 19 & $15,8 \%$ & Feminino & 64 & $53,3 \%$ \\
\hline 7 a 10 & 78 & $65,0 \%$ & Masculino & 56 & $46,7 \%$ \\
\hline 11 a 12 & 23 & $19,1 \%$ & Total & 120 & $100,0 \%$ \\
\hline Total & 120 & $100,0 \%$ & & & \\
\hline \multicolumn{3}{|c|}{ Crianças que tiveram COVID-19 } & \multicolumn{3}{|c|}{$\begin{array}{l}\text { Familiares ou pessoas próximas que } \\
\text { tiveram COVID-19 }\end{array}$} \\
\hline Sim & 06 & $5,0 \%$ & Sim & 51 & $42,5 \%$ \\
\hline Não & 107 & $89,2 \%$ & Não & 62 & $51,7 \%$ \\
\hline Não sabem & 07 & $5,8 \%$ & Não sabem & 07 & $5,8 \%$ \\
\hline Total & 120 & $100 \%$ & Total & 120 & $100 \%$ \\
\hline
\end{tabular}

Fonte: Bentinho e Katz (2022)

Tabela 2 - Cuidados com as crianças e comportamentos em relação à prevenção da COVID-19

\begin{tabular}{|c|c|c|c|c|c|}
\hline Variável & $\mathbf{n}$ & $\%$ & Variável & $\mathbf{n}$ & $\%$ \\
\hline \multicolumn{3}{|c|}{ Cumprimento do isolamento social } & \multicolumn{3}{|c|}{ Lavagem correta das mãos } \\
\hline $\operatorname{Sim}$ & 24 & $20,0 \%$ & $\operatorname{Sim}$ & 119 & $99,2 \%$ \\
\hline Não & 96 & $80,0 \%$ & Não & 01 & $0,8 \%$ \\
\hline Total & 120 & $100,0 \%$ & Total & 120 & $100,0 \%$ \\
\hline \multicolumn{3}{|c|}{ Comportamento/isolamento social $*$} & \multicolumn{3}{|c|}{ Máscara de uso exclusivo } \\
\hline Ficou em casa o tempo todo & 38 & $31,7 \%$ & $\operatorname{Sim}$ & 119 & $99,2 \%$ \\
\hline Supermercado/comércio/médico & 42 & $35,0 \%$ & Não & 01 & $0,8 \%$ \\
\hline Saiu para brincar na rua & 16 & $13,3 \%$ & Total & 120 & $100,0 \%$ \\
\hline Saiu para visitar um parente & 31 & $25,8 \%$ & & & \\
\hline \multicolumn{3}{|l|}{ Ações preventivas * } & \multicolumn{3}{|c|}{ Desconforto ao usar máscara } \\
\hline Sem alteração da rotina & 01 & $0,8 \%$ & Não & 65 & $54,2 \%$ \\
\hline Lavagem das mãos/álcool gel & 106 & $88,3 \%$ & Sim, mas usa & 53 & $44,2 \%$ \\
\hline Evitar tocar olhos, nariz e boca & 76 & $63,3 \%$ & Sim, por isso não usa & 02 & $1,6 \%$ \\
\hline Uso máscara ao sair de casa & 118 & $98,3 \%$ & Total & 120 & $100,0 \%$ \\
\hline Manter sapatos fora de casa & 73 & $60,8 \%$ & & & \\
\hline Etiqueta respiratória & 66 & $55,0 \%$ & & & \\
\hline
\end{tabular}

*Considerando que o respondente poderia selecionar mais de uma resposta

Fonte: Bentinho e Katz (2022)

A Tabela 2 mostra as respostas em relação aos cuidados com as crianças e comportamentos relacionado à prevenção da COVID-19. Sobre período de quarentena ou isolamento rígido, no qual era recomendado o isolamento social, oitenta por cento das crianças tiveram contato com outras pessoas além das que moravam no domicílio. A ida ao médico e ao supermercado/comércio junto com um responsável, foram os principais motivos para a saída da criança durante a quarentena. Das ações de prevenção ao COVID19 que mais fizeram parte da rotina das crianças, o uso de máscara ao sair de casa $(98,3 \%)$ 
e a lavagem das mãos frequentemente com água e sabão e/ou uso de álcool em gel $(88,3 \%)$, foram as principais. Quase a totalidade das crianças $(99,2 \%)$ foram ensinadas a fazer a lavagem correta das mãos. A grande maioria das crianças possuía máscaras exclusivas para seu uso $(99,2 \%)$ e um pouco mais da metade não relataram desconfortos ao usar máscaras $(54,2 \%)$.

$\mathrm{Na}$ Tabela 3 verifica-se que 90,0\% das crianças apresentaram mudança de comportamento no período do isolamento social. Os comportamentos mais relatados foram: tédio (46,8\%), ansiedade (44\%), irritação (40,4\%), agitação $(36,7 \%)$ e dificuldade para dormir (30,3\%). Sobre a percepção dos responsáveis da possibilidade de mudança de comportamento da criança durante o atendimento odontológico em decorrência das experiências vividas no período da pandemia, a maioria considerou que não haverá mudanças $(45,0 \%)$. Dos que consideraram que poderia haver mudanças, a maioria respondeu maior colaboração $(29,2 \%)$.

Tabela 3 - Distribuição da amostra em relação a mudanças de comportamento no período do isolamento social

\begin{tabular}{lcclcc}
\hline Variável & $\mathbf{n}$ & $\boldsymbol{\%}$ & Variável & $\mathbf{n}$ & $\%$ \\
\hline Mudança de comportamento da criança & & \multicolumn{4}{l}{ Mudança no comportamento nas consultas } \\
& & & odontológicas & & \\
Sim & 108 & $90,0 \%$ & Sim, mudanças positivas & 49 & $40,8 \%$ \\
Não & 12 & $10,0 \%$ & Sim, mudanças negativas & 17 & $14,2 \%$ \\
Total & 120 & $100,0 \%$ & Não & 54 & $45,0 \%$ \\
& & & Total & 120 & $100,0 \%$ \\
\hline Comportamentos relatados* & & & Tipo de mudança de comportamento* \\
Ansiedade & 48 & $44,0 \%$ & Não haverá mudanças & 54 & $45,0 \%$ \\
Medo & 23 & $21,1 \%$ & Maior colaboração & 35 & $29,2 \%$ \\
Depressão & 03 & $2,8 \%$ & Menor de colaboração & 06 & $5,0 \%$ \\
Tristeza & 30 & $27,5 \%$ & Ida mais frequente & 16 & $13,3 \%$ \\
Tédio & 51 & $46,8 \%$ & Medo/ansiedade & 11 & $9,2 \%$ \\
Agitação/Hiperatividade & 40 & $36,7 \%$ & Outros & 01 & $0,8 \%$ \\
Irritação & 44 & $40,4 \%$ & & & \\
Dificuldade para dormir & 33 & $30,3 \%$ & & & \\
Dificuldade de concentração & 27 & $24,8 \%$ & & \\
\hline
\end{tabular}

*Considerando que o respondente poderia selecionar mais de uma resposta Fonte: Bentinho e Katz (2022)

A Tabela 4 mostra que $83,3 \%$ das crianças mudaram seus hábitos alimentares durante a pandemia, passando a comer mais $(71,1 \%)$. A maioria dos responsáveis $(70,0 \%)$ relatou que houve redução da renda familiar, e para $16,7 \%$, esta redução da renda teve impacto na alimentação da criança. Grande parte da amostra $(75,0 \%)$ teve alterações nos hábitos de higiene bucal (Tabela 5). 
Tabela 4 - Mudanças nos hábitos alimentares das crianças durante a pandemia e impacto da renda familiar

\begin{tabular}{|c|c|c|c|c|c|}
\hline Variável & $\mathbf{n}$ & $\%$ & Variável & $\mathbf{n}$ & $\%$ \\
\hline \multicolumn{3}{|c|}{ Mudança dos hábitos alimentares } & \multicolumn{3}{|l|}{ Redução da renda familiar } \\
\hline Sim & 100 & $83,3 \%$ & $\operatorname{Sim}$ & 84 & $70,0 \%$ \\
\hline Não & 20 & $16,7 \%$ & Não & 36 & $30,0 \%$ \\
\hline Total & 120 & $100 \%$ & Total & 120 & $100,0 \%$ \\
\hline \multicolumn{3}{|l|}{ Mudanças relatadas* } & \multicolumn{3}{|c|}{ Impacto da redução da renda na alimentação } \\
\hline Não apresentou mudança & 20 & $16,7 \%$ & $\operatorname{Sim}$ & 20 & $16,7 \%$ \\
\hline Passou a comer mais & 86 & $71,7 \%$ & Não & 64 & $53,3 \%$ \\
\hline Passou a comer menos & 09 & $7,5 \%$ & Não houve redução da renda & 36 & $30,0 \%$ \\
\hline $\begin{array}{l}\text { Passou a comer mais comidas } \\
\text { feitas em casa }\end{array}$ & 53 & $44,2 \%$ & Total & 120 & $100,0 \%$ \\
\hline $\begin{array}{l}\text { Passou a comer enlatados, } \\
\text { conservas ou industrializados }\end{array}$ & 02 & $1,7 \%$ & & & \\
\hline $\begin{array}{l}\text { Passou a comer mais lanches, } \\
\text { bolachas, doces, salgadinhos }\end{array}$ & 37 & $30,8 \%$ & & & \\
\hline
\end{tabular}

*Considerando que o respondente poderia selecionar mais de uma resposta

Fonte: Bentinho e Katz (2022)

Tabela 5 - Distribuição da amostra de acordo com a rotina de higiene bucal da criança durante a pandemia

\begin{tabular}{lcc}
\hline Variável & Número & Percentual \\
\hline Houve mudança de hábitos de higiene bucal? & & \\
Sim & 90 & $75,0 \%$ \\
Não & 30 & $25,0 \%$ \\
Total & 120 & $100,0 \%$ \\
& & \\
Mudanças na higiene bucal* & & \\
Não houve alterações & 30 & $25 \%$ \\
Esqueceu de escovar os dentes algumas vezes & 43 & $35,8 \%$ \\
Dormiu sem escovar os dentes & 25 & $20,8 \%$ \\
Passou a escovar os dentes mais vezes & 27 & $22,5 \%$ \\
\hline
\end{tabular}

*Considerando que o respondente poderia selecionar mais de uma resposta

Fonte: Bentinho e Katz (2022)

A Tabela 6 mostra que 30,8\% das crianças sentiram dor de dente durante a quarentena, $35,8 \%$ tiveram necessidade de atendimento odontológico e $29,2 \%$ foram atendidas pelo dentista. Os serviços de atendimento mais procurados foram, respectivamente, particular (51,4\%), público de urgência ou Unidade de Pronto Atendimento-UPA $(25,7 \%)$, convênios $(14,3 \%)$ e postos de saúde públicos $(8,6 \%)$. 
Tabela 6 - Dados referentes à saúde bucal das crianças durante o período da pandemia, de acordo com a percepção dos responsáveis

\begin{tabular}{lcclccc}
\hline Variável & n & \multirow{2}{*}{ Variável } & n & $\%$ \\
\hline A criança sentiu dor de dente? & & & A criança foi atendida por um dentista? \\
Sim & 37 & $30,8 \%$ & Sim & 35 & $29,2 \%$ \\
Não & 83 & $69,2 \%$ & Não & 85 & $70,8 \%$ \\
Total & 120 & $100,0 \%$ & Total & 120 & $100,0 \%$ \\
\hline A criança precisou ir ao dentista? & & & Serviço em que a criança foi atendida* \\
Sim & 43 & $35,8 \%$ & Particular & 18 & $51,4 \%$ \\
Não & 77 & $64,2 \%$ & Convênio & 05 & $14,3 \%$ \\
Total & 120 & $100,0 \%$ & Urgência ou UPA & 09 & $25,7 \%$ \\
& & & Posto de saúde & 03 & $8,6 \%$ \\
& & & Total & 35 & $29.2 \%$ \\
\hline
\end{tabular}

\section{Motivo da ida ao dentista}

Cárie/dor/necessidade de extração $27 \quad 77,1 \%$

Trauma $02 \quad 5,7 \%$

Problemas com aparelho $\quad 01 \quad 2,8 \%$

Restauração fraturada $\quad 01 \quad 2,8 \%$

Consultas de rotina $\quad 04 \quad 11,4 \%$

Total $35 \quad 100,0 \%$

*Considerando que o respondente poderia selecionar mais de uma resposta

Fonte: Bentinho e Katz (2022)

\section{DISCUSSÃO}

Nesta pesquisa, de acordo com o relato dos pais/responsáveis, o percentual de crianças acometidas pelo novo coronavírus foi muito baixo. Apesar do fato de que este número pode não corresponder à realidade, uma vez que as crianças não foram testadas e/ou podem ter ficado assintomáticas, este dado corrobora com os estudos que indicaram que as crianças são um dos grupos que menos sofrem com a doença (DONG, et al. 2020; LU, et al. 2020). Por outro lado, foi possível observar um percentual significativo das crianças com familiares e amigos próximos que desenvolveram a COVID-19.

Apesar da importância do distanciamento social para diminuir a propagação da doença, a pesquisa revelou que um percentual significativo de crianças não teve restrição de contato com pessoas externas ao domicílio. Este resultado mostra a dificuldade da população na adesão das medidas restritivas e a falta de informação a respeito de que as crianças podem ser vetores da doença (DONG, et al. 2020).

Com relação às ações preventivas, os resultados desta pesquisa foram semelhantes àquela realizada com adolescentes de 12 a 15 anos pela Fundação Oswaldo Cruz (2020) em que os comportamentos de prevenção mais frequentes foram o uso de máscaras, lavagem das mãos com água em sabão, e/ou uso de álcool em gel. Porém, ao 
compararmos a margem percentual das duas pesquisas, este estudo mostra que as crianças se apresentam mais atuantes nas ações de prevenção à COVID-19 que os adolescentes.

$\mathrm{Na}$ pesquisa, quase a totalidade das crianças foram ensinadas a lavar as mãos corretamente por algum familiar na pandemia, o que corrobora com Chen et al. (2020) ao afirmarem a importância dos pais em fazer pleno uso do período de isolamento social para ensinar as crianças a desenvolverem uma boa consciência pessoal de higiene, bem como de instruí-las a manter um comportamento de lavagem frequente das mãos.

Diferente do estudo de Aronu et al. (2020), em que, segundo as mães, menos da metade dos seus filhos utilizavam máscaras como forma de prevenção ao COVID-19, quase que a totalidade das crianças deste estudo fizeram uso das máscaras ao saírem de casa, tendo esses itens para seu uso exclusivo. Apesar de um pouco mais da metade das crianças deste estudo não apresentarem problemas na utilização das máscaras de proteção, um percentual considerável relatou desconforto, revelando a dificuldade da população em se acostumar com essa nova rotina de proteção. Este desconforto pode estar relacionado à idade, pois crianças menores podem apresentar dificuldade para respirar e utilizar por tempo prolongado (ARONU, et al. 2020; ESPOSITO e PRINCIPI, 2020); como também, pode estar relacionado ao tamanho ou material inadequados. Neste estudo, apenas duas crianças (de 3 anos de idade) não faziam uso de máscaras, sendo o desconforto a justificativa apresentada.

Existem divergências sobre a partir de qual idade é recomendado o uso de máscaras por crianças. A Organização Mundial da Saúde (WHO, UNICEF, 2020) recomenda que não deve haver obrigatoriedade do uso de máscaras em menores de 5 anos. No entanto, alguns países podem considerar o uso de máscaras de proteção para crianças acima de 2 ou 3 anos. No Brasil, a Sociedade Brasileira de Pediatria (2020), recomendou a utilização de máscaras em crianças a partir dos 2 anos, com orientação e monitoramento dos pais. Assim, é importante que em crianças menores, na faixa etária de 2 a 6 anos, haja uma supervisão constante dos pais para garantir o uso correto da máscara e para prevenir qualquer dano potencial associado ao uso desse item de proteção pela criança (SBP, 2020).

Observou-se que a grande maioria das crianças apresentou alguma alteração de comportamento durante a pandemia. Da mesma forma que encontrado em outros estudos, os comportamentos mais frequentes foram o tédio (ORGILÉS, et al. 2020), ansiedade (JIAO, et al. 2020; XIE, et al. 2020), irritação (JIAO, et al. 2020; ORGILÉS, et al. 2020; 
ROCHA, et al. 2020), agitação (JIAO, et al. 2020; ROCHA, et al. 2020), dificuldade para dormir (JIAO, et al. 2020; ROCHA, et al. 2020), dificuldade de concentração (JIAO, et al. 2020; ORGILÉS, et al. 2020), hiperatividade e medo (JIAO, et al. 2020). Assim como observado por Xie et al. (2020), a privação de atividades ao ar livre, da ida à escola e da socialização, tão importantes para crianças que estão em desenvolvimento, pode resultar em alterações de comportamento.

Nesta pesquisa, metade das crianças apresentaram tédio, assim como um elevado percentual passou a comer mais, confirmando que o confinamento pode gerar alterações de comportamento, as quais também podem estar relacionadas a alterações alimentares (MOYNIHAN, et al. 2015). Observou-se um percentual muito elevado de crianças que passaram a comer mais e, não necessariamente, alimentos saudáveis. Um pequeno percentual de responsáveis relatou sintomas de depressão nas crianças $(2,8 \%)$, entretanto, quando se considera os relatos de tristeza, como um sintoma relacionado à depressão, este percentual é cerca de $30 \%$. Esses dados podem representar demandas futura para os profissionais que cuidam da saúde física e mental infantil.

Associando o comportamento infantil com a Odontologia, a maioria dos respondentes acreditam que haverá mudança no comportamento infantil durante o atendimento odontológico. Ao contrário do esperado, em meio aos impactos emocionais da pandemia de COVID-19, os resultados mostram-se animadores para a área da Odontopediatria, uma vez que houve um elevado percentual de responsáveis que consideraram que os comportamentos das crianças nas próximas consultas serão positivos, com maior colaboração e visitas mais frequentes.

Estudos têm mostrado que a pandemia tem elevado o nível de ansiedade nas crianças (JIAO, et al. 2020; XIE, et. al. 2020). O estudo de Olszewska e Rzymski (2020), que comparou os níveis de ansiedade ao tratamento odontológico em 25 crianças polonesas na faixa etária de 7 anos, atendidas antes e durante a pandemia, não revelou diferenças estatisticamente significantes. As crianças que foram ao dentista durante a quarentena nacional não apresentaram um nível de ansiedade significativamente mais alto em comparação com o grupo de controle pré-pandêmico, independentemente de seu estado emocional ter sido avaliado pelo dentista, pelo cuidador ou pelas próprias crianças. Considerando uma possível segunda onda da pandemia em vários países, paralisando novamente os atendimentos odontológicos, sugere-se que a relação entre as alterações 
comportamentais vinculadas à situação da pandemia e o manejo comportamental das crianças no atendimento odontológico sejam temas de pesquisas futuras.

A nova rotina trazida pela pandemia, o trabalho em casa para os pais, a aulas remotas para os filhos e instabilidade econômica contribuíram para a mudanças nos hábitos alimentares (DI RENZO, et al. 2020). Concordando com os estudos de Campagnaro et al. (2020) e Carroll et al. (2020), a grande maioria das crianças apresentou alterações nos hábitos alimentares durante a pandemia, passando a comer mais. Embora um elevado percentual tenha passado a comer mais comidas caseiras, comparativamente, também houve um elevado percentual de crianças que passaram a comer mais lanches (fast food), doces, comidas industrializadas. Assim, observa-se a formação de dois grupos distintos: o das crianças que podem ter recebido maior atenção dos pais, durante o período de isolamento social, em relação à alimentação saudável, feita em casa; e o grupo daquelas que podem não ter tido esse cuidado, ou passaram a consumir alimentos menos saudáveis por serem de fácil acesso e menor custo. Desta forma, novas pesquisas poderão ser realizadas com foco nestas variáveis a fim de esclarecer melhor esses eventos.

Uma variável que pode interferir na alimentação é diminuição da renda das famílias. Neste estudo, a grande maioria teve redução da renda familiar, assim como também encontrado por Campagnaro et al. (2020). Por outro lado, metade dos respondentes afirmaram que esta redução não produziu impacto na alimentação das crianças. No entanto, é importante não desconsiderar o percentual de quase vinte por cento que relatou haver impacto. Futuros estudos com amostras maiores serão oportunos, uma vez esta variável poderá sofrer mudanças, a depender do tempo de prolongamento da pandemia.

Considerando os hábitos de higiene bucal, os resultados foram alarmantes: mais de setenta por cento das crianças mostraram mudanças significativas em seus hábitos de higiene bucal. Em concordância com outros estudos (CAMPAGNARO, et al. 2020; BRESCIA, et al. 2021; LIU, et al. 2021), esses achados, associados às alterações alimentares, anunciam as próximas demandas da área da Odontologia.

Em reforço a este raciocínio, pode-se considerar os resultados elevados em relação às crianças que sentiram dor de dente (30\%). Este dado foi próximo ao encontrado em estudo realizado por Liu et al. (2021) com crianças na cidade de Wuhan, epicentro no início da epidemia, durante o lockdown. 
Mesmo com a limitações nos atendimentos clínicos odontológicos durante a pandemia da COVID-19, das crianças que precisaram de atendimento, grande parte foi foi atendida por um dentista. Estes resultados divergem dos estudos de Campagnaro et al. (2020) e Sun et al. (2020) que afirmaram que apesar da necessidade, muitos pais não iriam ao dentista devido ao medo da infecção pelo COVID-19 no ambiente odontológico.

Das crianças que foram atendidas, um pouco mais da metade recorreram ao serviço clínico particular, o que pode estar associado a uma maior dificuldade de conseguir atendimento, mesmo que de urgência, no serviço público de saúde em meio à pandemia. Esse é um dado crítico, haja vista o impacto da pandemia na renda das famílias, considerando que grande parte dos pacientes das clínicas odontológicas da Universidade não têm boas condições financeiras, sendo a gratuidade dos serviços odontológicos das clínicas-escolas um dos motivos de procura do atendimento que é oferecido.

Considerando os motivos mais relatados à procura do atendimento odontológico durante a pandemia (cárie, dor, traumas, problemas com aparelhos ortodônticos), estes concordaram com os encontrados por Liu et al. (2021) e Yang et al. (2021) em seus estudos realizados com crianças na cidade de Wuhan. É importante salientar que a falta de cuidados preventivos e curativos pode impactar negativamente na saúde bucal das crianças, já que a pandemia continua, e mesmo após mais de um ano, alguns serviços de saúde - principalmente os públicos - não normalizaram seus atendimentos. Conhecer a percepção dos pais/responsáveis sobre a alimentação e a saúde bucal das crianças é importante, uma vez que suas decisões têm impacto no bem-estar infantil. Os resultados desta pesquisa são úteis para a elaboração de estratégias de cuidado à saúde da população infantil, incluindo ações para auxiliar os pais a melhorarem os cuidados na alimentação e higiene, bem como preparar as equipes de saúde para as demandas que virão no póspandemia.

\section{CONCLUSÕES}

O período de isolamento social em 2020, como parte das medidas necessárias ao combate à pandemia da COVID-19, implicou em mudanças comportamentais, de hábitos alimentares e de higiene bucal para grande maioria da amostra estudada. As alterações comportamentais mais observadas foram ansiedade, tédio e irritabilidade. Quanto às rotinas alimentares, as crianças passaram a comer mais, com percentuais próximos, tanto 
para as refeições caseiras, quanto para as consideradas não saudáveis. As queixas odontológicas mais frequentes foram cárie, necessidade de extrações dentárias e dor.

Os resultados encontrados merecem atenção dos profissionais de saúde na preparação das estratégias de atendimento às novas demandas de saúde relacionadas ao prolongamento do período da pandemia.

\section{REFERÊNCIAS}

AQUINO, E. M. L. et al. Medidas de distanciamento social no controle da pandemia de COVID-19: potenciais impactos e desafios no Brasil. Ciência e Saúde Coletiva, v. 25, supl. 1, p. 2423-2446, 2020.

ARONU, A. E. et al. Maternal perception of masking in children as a preventive strategy for COVID-19 in Nigeria: A multicentre study. PLoS One, v. 15, n. 11, p. e0242650, 2020.

SOCIEDADE BRASILEIRA DE PEDIATRIA. O uso de máscaras faciais em tempo de COVID-19 por crianças e adolescentes: Uma proposta inicial. Disponível em: https://www.sbp.com.br/fileadmin/user_upload/22543e-NA__O_uso_mascaras_faciais_em_COVID19_por_crc_e_adl_1_.pdf

BRESCIA, A.V. et al. Impact of lockdown on children's lifestyle and their collaboration during dental sessions. European Journal of Paediatric Dentistry, v. 22, n. 1, p. 61$65,2021$.

CAMPAGNARO, R. et al. COVID-19 pandemic and pediatric dentistry: Fear, eating habits and parent's oral health perceptions. Children and Youth Services Review, v. 118, p. e105469, 2020.

CARROLL, N. et al. The Impact of COVID-19 on health behavior, stress, financial and food security among middle to high income Canadian families with young children.

Nutrients, v.12, n. 8, p. 2352, 2020.

CHEN, X. et al. Hand Hygiene, mask-wearing behaviors and its associated factors during the COVID-19 Epidemic: A cross-sectional study among primary school students in Wuhan, China. International Journal of Environmental Research and Public Health, v. 17, n. 8, p. 2893, 2020.

CHISINI L. A. et al. COVID-19 pandemic impact on paediatric dentistry treatments in the Brazilian Public Health System. International Journal of Paediatric Dentistry, v. 31, n. 1, p. 31-34, 2021.

FUNDAÇÃO OSWALDO CRUZ. ConVid Adolescentes - Pesquisa de Comportamento. Fundação Oswaldo Cruz. 2020. Disponível em: https://convid.fiocruz.br/index.php?pag=comportamentos_adolescentes.

DI RENZO L, et al. Eating habits and lifestyle changes during COVID-19 lockdown: an Italian survey. Journal of Translational Medicine, v. 18, n. 1, p. 229, 2020.

DONG Y, et al. Epidemiology of COVID-19 among children in China. Pediatrics, v. 145, n. 6, p. e20200702, 2020. 
ESPOSITO, S.; PRINCIPI, N. To mask or not to mask children to overcome COVID19. European Journal of Pediatrics, v.179, n. 8, p. 1267-1270, 2020.

JIAO, W. Y. et al. Behavioral and Emotional Disorders in Children during the COVID19 Epidemic. Journal of Pediatrics, v. 221, p. 264-266.e1, 2020.

LIU, C. et al. The impact of coronavirus lockdown on oral healthcare and its associated issues of pre-schoolers in China: an online cross-sectional survey. BMC Oral Health, v. 21, n. 1, p. 54, 2021.

LU, X. et al. SARS-CoV-2 Infection in Children. The New England Journal of Medicine, v. 382, n. 17, p. 1663-1665, 2020.

LUZZI, V. et al. Paediatric Oral Health during and after the COVID-19 pandemic. International Journal of Paediatric Dentistry, v. 31, n.1, p. 20-26.

MOYNIHAN, A. B. et al. Eaten up by boredom: consuming food to escape awareness of the bored self. Frontiers in Psychology, v. 1, n. 6, p. 369, 2015.

OLSZEWSKA, A.; RZYMSKI, P. children's dental anxiety during the COVID-19 pandemic: Polish experience. Journal of Clinical Medicine, v.9, n. 9, p. 2751, 2020.

ORGILÉS, M. et al. Immediate psychological effects of the COVID-19 quarantine in youth from Italy and Spain. Frontiers in Psychology. v. 6, n. 11, p. 579038, 2020.

ROCHA, M. F. A. et al. The impact of the covid-19 pandemic on child-youth health: a cross-sectional study. O impacto da pandemia do covid-19 na saúde infanto-juvenil: um estudo transversal. Brazilian Journal of Health Review, v. 4, n. 1, p. 3483-3497, 2020.

SUN, J. et al. Knowledge of and attitudes toward COVID-19 among parents of child dental patients during the outbreak. Brazilian Oral Reserch. v. 34, p. e066, 2020.

ORGANIZAÇÃO MUNDIAL DE SAÚDE. WHO Director-General's opening remarks at the media briefing on COVID-19 - 11 March 2020. Disponivel em: https://www.who.int/director-general/speeches/detail/who-director-general-s-openingremarks-at-the-media-briefing-on-covid-19---11-march-2020

WHO, UNICEF. World Health Organization and UNICEF. Advice on the use of masks for children in the community in the context of COVID-19. 2020. Disponível em: https://apps.who.int/iris/bitstream/handle/10665/333919/WHO-2019-nCoVIPC_Masks-Children-2020.1-eng.pdf?sequence $=1 \&$ isAllowed $=y$

XIE, X. et al. Mental health status among children in home confinement during the coronavirus disease 2019 outbreak in Hubei Province, China. JAMA Pediatrics, v. 174, n. 9, p. 898-900, 2020.

YANG, F. et al. Online consultation and emergency management in paediatric dentistry during the COVID-19 epidemic in Wuhan: A retrospective study. International Journal of Paediatric Dentistry, v. 31, n. 1, p. 5-11. 2021.

Recebido em: 15/01/2022

Aprovado em: 16/02/2022

Publicado em: 18/02/2022 